\title{
EXISTENCE OF SOLUTIONS FOR A NONLINEAR ELLIPTIC DIRICHLET BOUNDARY VALUE PROBLEM WITH AN INVERSE SQUARE POTENTIAL
}

\author{
SHENGHUA WENG AND YONGQING LI
}

Received 11 January 2006; Accepted 24 March 2006

Via the linking theorem, the existence of nontrivial solutions for a nonlinear elliptic Dirichlet boundary value problem with an inverse square potential is proved.

Copyright (C) 2006 S. Weng and Y. Li. This is an open access article distributed under the Creative Commons Attribution License, which permits unrestricted use, distribution, and reproduction in any medium, provided the original work is properly cited.

\section{Introduction}

This paper is concerned with the existence of nontrivial solutions to the following problem:

$$
\begin{gathered}
-\triangle u-\frac{\mu}{|x|^{2}} u=|u|^{p-2} u+\lambda u \quad \text { in } \Omega \backslash\{0\}, \\
u(x)=0 \quad \text { on } \partial \Omega,
\end{gathered}
$$

where $0 \in \Omega \subset \mathbb{R}^{N}(N \geq 3)$ is a bounded domain with smooth boundary, $0 \leq \mu<\bar{\mu}=$ $((N-2) / 2)^{2}$, and $\bar{\mu}$ is the best constant in the Hardy inequality:

$$
C \int_{\mathbb{R}^{N}} \frac{u^{2}}{|x|^{2}} d x \leq \int_{\mathbb{R}^{N}}|\nabla u|^{2} d x
$$

(cf. [3, Lemma 2.1]), $2<p<2^{*}$, where $2^{*}=2 N /(N-2)$ is the so-called critical Sobolev exponent and $\lambda>0$ is a parameter.

Finally, in Theorem 1.3 we prove, for small $\lambda>0$, the existence of a solution to

$$
\begin{aligned}
-\Delta u-\frac{\mu}{|x|^{2}} u & =u^{p-1}+\lambda u \quad \text { in } \Omega \backslash\{0\}, \\
u(x)>0 & \text { in } \Omega \backslash\{0\}, \\
u(x)=0 & \text { on } \partial \Omega .
\end{aligned}
$$


In the case $\mu=0$, problem (1.1) has been studied extensively. For example, when $p=$ $2^{*}$, Capozzi et al. [1] have shown that (1.1) has at least one positive solution for $N \geq$ 5. When $2<p<2^{*}$, the existence of positive solutions of (1.1) has been shown in [5, Chapter 1].

Our results are the following.

Theorem 1.1. Let $0 \in \Omega \subset \mathbb{R}^{N}(N \geq 3)$ be an open bounded domain. If $0 \leq \mu<\bar{\mu}$, then for any $\lambda>0$, problem (1.1) possesses a nontrivial solution.

Remark 1.2. We mention that when $p=2^{*}$, the existence of nontrivial solutions of (1.1) has been proved in [2, Theorem 1.3].

Theorem 1.3. Let $0 \in \Omega \subset \mathbb{R}^{N}(N \geq 3)$ be an open bounded domain. If $0 \leq \mu<\bar{\mu}$, problem (1.3) has a positive solution for $0<\lambda<\lambda_{1}$, where $\lambda_{1}$ denotes the first eigenvalue of the operator $-\triangle-\mu /|x|^{2}$.

This paper is organized as follows. In Section 2, we give some preliminaries. Section 3 is devoted to the proof of Theorem 1.1. The proof of Theorem 1.3 is contained in Section 4.

\section{Notations and preliminaries}

Throughout this paper, $c, c_{i}$ will denote various positive constants whose exact values are not important. $H_{0}^{1}(\Omega)$ will be denoted as the standard Sobolev space, whose norm $\|\cdot\|$ is deduced by the standard inner product. By $|\cdot|_{p}$, we denote the norm of $L^{p}(\Omega)$. All integrals are taken over $\Omega$ unless stated otherwise. On $H_{0}^{1}(\Omega)$, we use the norm

$$
\|u\|_{\mu}^{2}=\int\left(|\nabla u|^{2}-\frac{\mu}{|x|^{2}} u^{2}\right) d x .
$$

It follows from the Hardy inequality that the norm $\|\cdot\|_{\mu}$ is equivalent to the usual norm $\|\cdot\|$ of $H_{0}^{1}(\Omega) . H_{0}^{1}(\Omega)$ with the norm $\|\cdot\|_{\mu}$ is simply denoted by $H$.

By using the critical point theory, we define the action function on $H$ :

$$
J_{\mu}(u)=\frac{1}{2} \int\left(|\nabla u|^{2}-\frac{\mu}{|x|^{2}} u^{2}\right) d x-\frac{1}{p} \int|u|^{p} d x-\frac{\lambda}{2} \int|u|^{2} d x
$$

It is well known that a weak solution $u \in H_{0}^{1}(\Omega)$ of $(1.1)$ is precisely a critical point of $J_{\mu}$. That is,

$$
\left\langle J_{\mu}^{\prime}(u), \varphi\right\rangle=\int\left(\nabla u \nabla \varphi-\frac{\mu}{|x|^{2}} u \varphi\right) d x-\int|u|^{p-2} u \varphi d x-\lambda \int u \varphi d x=0
$$

holds for any $\varphi \in H_{0}^{1}(\Omega)$. The following definition has become standard. 
Definition 2.1 (see [6, Definition 1.16]). Let $c \in \mathbb{R}$, let $E$ be a Banach space, and let $I \in$ $C^{1}(E, \mathbb{R})$. Say that $I$ satisfies $(\mathrm{PS})_{c}$ condition if any sequence $\left\{u_{n}\right\}$ in $E$ such that $I\left(u_{n}\right) \rightarrow c$ and $\left\|I^{\prime}\left(u_{n}\right)\right\|_{E^{-1}} \rightarrow 0$ has a convergent subsequence. If this holds for every $c \in \mathbb{R}, I$ satisfies (PS) condition.

Now we will prove that $J_{\mu}$ satisfies (PS) condition, which is contained in the following two lemmas.

LEMma 2.2. If $0 \leq \mu<\bar{\mu}=((N-2) / 2)^{2}$, then any sequence $\left\{u_{n}\right\} \subset H_{0}^{1}(\Omega)$ satisfying

$$
J_{\mu}\left(u_{n}\right) \longrightarrow c, \quad J_{\mu}^{\prime}\left(u_{n}\right) \longrightarrow 0, \quad n \longrightarrow \infty,
$$

is bounded in $H_{0}^{1}(\Omega)$.

Proof. Since

$$
\begin{gathered}
J_{\mu}\left(u_{n}\right)=\frac{1}{2} \int\left(\left|\nabla u_{n}\right|^{2}-\frac{\mu}{|x|^{2}} u_{n}^{2}\right) d x-\frac{1}{p} \int\left|u_{n}\right|^{p} d x-\frac{\lambda}{2} \int\left|u_{n}\right|^{2} d x, \\
\left\langle J_{\mu}^{\prime}\left(u_{n}\right), \varphi\right\rangle=\int\left(\nabla u_{n} \nabla \varphi-\frac{\mu}{|x|^{2}} u_{n} \varphi\right) d x-\int\left|u_{n}\right|^{p-2} u_{n} \varphi d x-\lambda \int u_{n} \varphi d x .
\end{gathered}
$$

Choose $2<q<p$, and let $\varphi=u_{n}$ in (2.5). For $n$ large enough,

$$
\begin{aligned}
c+1 & +o(1)\left\|u_{n}\right\|_{\mu} \\
& \geq J_{\mu}\left(u_{n}\right)-\frac{1}{q}\left\langle J_{\mu}^{\prime}\left(u_{n}\right), u_{n}\right\rangle \\
& =\left(\frac{1}{2}-\frac{1}{q}\right)\left\|u_{n}\right\|_{\mu}^{2}+\left(\frac{1}{q}-\frac{1}{p}\right) \int\left|u_{n}\right|^{p} d x+\left(\frac{1}{q}-\frac{1}{2}\right) \lambda \int\left|u_{n}\right|^{2} d x \\
& \geq\left(\frac{1}{2}-\frac{1}{q}\right)\left\|u_{n}\right\|_{\mu}^{2}+\left(\frac{1}{q}-\frac{1}{p}\right) \int\left|u_{n}\right|^{p} d x+\left(\frac{1}{q}-\frac{1}{2}\right) \lambda C\left\|u_{n}\right\|_{\mu}^{2} .
\end{aligned}
$$

It follows from $p>2$ that $\left\{u_{n}\right\}$ is bounded in $H_{0}^{1}(\Omega)$.

LEMMA 2.3. Under the assumption of Lemma 2.2, $\left\{u_{n}\right\}$ possesses a convergent subsequence in $H$.

Proof. By Lemma 2.2, going if necessary to a subsequence, we can assume that

$$
\begin{aligned}
& u_{n} \rightarrow u \quad \text { in } H, \\
& u_{n} \longrightarrow u \text { in } L^{r}(\Omega) \text { for } 1 \leq r<2^{*} .
\end{aligned}
$$

Let $f(u)=|u|^{p-2} u$, [5, Theorem A.2] implies that $f\left(u_{n}\right) \rightarrow f(u)$ in $L^{s}$, where $s=r /(r-$ 1). Observe that

$$
\left\|u_{n}-u\right\|_{\mu}^{2}=\left\langle J_{\mu}^{\prime}\left(u_{n}\right)-J_{\mu}^{\prime}(u), u_{n}-u\right\rangle+\int\left[\left(f\left(u_{n}\right)-f(u)\right)\left(u_{n}-u\right)+\lambda\left(u_{n}-u\right)^{2}\right] d x .
$$


4 Solutions for a nonlinear elliptic Dirichlet BVP

It is clear that

$$
\left\langle J_{\mu}^{\prime}\left(u_{n}\right)-J_{\mu}^{\prime}(u), u_{n}-u\right\rangle \longrightarrow 0, \quad n \longrightarrow \infty .
$$

It follows from the Hölder inequality that

$$
\int\left[\left(f\left(u_{n}\right)-f(u)\right)\left(u_{n}-u\right)\right] d x \leq\left|f\left(u_{n}\right)-f(u)\right|_{r /(r-1)}\left|u_{n}-u\right|_{r} \rightarrow 0, \quad n \longrightarrow \infty .
$$

Thus we have proved that $\left\|u_{n}-u\right\|_{\mu} \rightarrow 0, n \rightarrow \infty$.

\section{Proof of Theorem 1.1}

In this section, we will prove Theorem 1.1 via the following linking theorem from Rabinowitz [5, Theorem 5.3] (see also [6]).

Proposition 3.1. Let E be a Banach space with $E=Y \oplus X$, where $\operatorname{dim} Y<\infty$. Suppose that $I \in C^{1}(E, \mathbb{R})$ and satisfies that

(i) there exist $\rho, \alpha>0$ such that $\left.I\right|_{\partial B_{\rho} \cap X} \geq \alpha$;

(ii) there exist $e \in \partial B_{1} \cap X$ and $R>\rho$ such that if $Q \equiv\left(\overline{B_{\rho}} \cap Y\right) \oplus\{$ re $; 0<r<R\}$, then $\left.I\right|_{\partial Q} \leq 0$.

If I satisfies $(P S)_{c}$ condition with

$$
c=\inf _{h \in \Gamma} \max _{u \in Q} I(h(u)),
$$

where

$$
\Gamma=\left\{h \in C(\bar{Q}, E) ;\left.h\right|_{\partial Q}=\mathrm{id}\right\},
$$

then $c$ is a critical value of $I$ and $c \geq \alpha$.

Remark 3.2 (see [5, Remark 5.5(iii)]). Suppose $\left.I\right|_{Y} \leq 0$ and there are an $e \in \partial B_{1} \cap X$ and $\widetilde{T}>\rho$ such that $I(u) \leq 0$ for $u \in Y \oplus \operatorname{span}\{e\}$ and $\|u\| \geq \widetilde{T}$, then for any large $T, Q=$ $\left(\overline{B_{\rho}} \cap Y\right) \oplus\{$ te $; 0<t<T\}$ satisfies $\left.I\right|_{\partial Q} \leq 0$.

To continue our discussion, we may assume that there is $k$ such that $\lambda_{k} \leq \lambda<\lambda_{k+1}$, where $\lambda_{k}$ is the $k$ th eigenvalue of the operator $\left(-\Delta-\mu /|x|^{2}\right)$ with Dirichlet boundary condition (see $[2,4])$. Let

$$
Y:=Y_{k}=\operatorname{span}\left\{\phi_{1}, \phi_{2}, \ldots, \phi_{k}\right\}
$$

here $\phi_{i}$ denotes the eigenfunction corresponding to $\lambda_{i}$. Decompose $H_{0}^{1}(\Omega)=Y \oplus X$ (where $X$ is the topological complement of $Y$ in $\left.H_{0}^{1}(\Omega)\right)$. For any $y \in Y$, we have that

$$
\begin{gathered}
\int\left(|\nabla y|^{2}-\frac{\mu}{|x|^{2}} y^{2}\right) d x \leq \lambda_{k} \int y^{2} d x \\
\int\left(|\nabla u|^{2}-\frac{\mu}{|x|^{2}} u^{2}\right) d x \geq \lambda_{k+1} \int u^{2} d x \quad \text { for any } u \in X .
\end{gathered}
$$

Now we will show that $J_{\mu}$ satisfies (i), (ii) in Proposition 3.1 in our situation. 
Proposition 3.3. There exist $\rho, \alpha>0$ such that $J_{\mu} \mid \partial B_{\rho} \cap X \geq \alpha$.

Proof. For any $u \in X, \lambda_{k} \leq \lambda<\lambda_{k+1}$, we obtain from (3.5) and Sobolev inequality that

$$
\begin{aligned}
J_{\mu}(u) & =\frac{1}{2} \int\left(|\nabla u|^{2}-\frac{\mu}{|x|^{2}} u^{2}\right) d x-\frac{1}{p} \int|u|^{p} d x-\frac{\lambda}{2} \int|u|^{2} d x \\
& \geq \frac{1}{2} \frac{\lambda_{k+1}-\lambda}{\lambda_{k+1}} \int\left(|\nabla u|^{2}-\frac{\mu}{|x|^{2}} u^{2}\right) d x-\frac{1}{p} \int|u|^{p} d x \\
& \geq \frac{1}{2} \frac{\lambda_{k+1}-\lambda}{\lambda_{k+1}}\|u\|_{\mu}^{2}-c\|u\|_{\mu}^{p} .
\end{aligned}
$$

Then we can choose $\|u\|_{\mu}=\rho$ sufficiently small and $\alpha>0$ such that $\left.J_{\mu}\right|_{\partial B_{\rho} \cap X} \geq \alpha$.

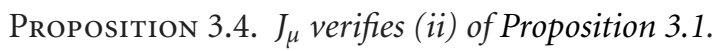

Proof. First, for any $y \in Y$, we obtain from (3.4) that

$$
\begin{aligned}
J_{\mu}(y) & =\frac{1}{2} \int\left(|\nabla y|^{2}-\frac{\mu}{|x|^{2}} y^{2}\right) d x-\frac{1}{p} \int|y|^{p} d x-\frac{\lambda}{2} \int|y|^{2} d x \\
& \leq \frac{1}{2} \frac{\lambda_{k}-\lambda}{\lambda_{k}} \int\left(|\nabla y|^{2}-\frac{\mu}{|x|^{2}} y^{2}\right) d x-\frac{1}{p} \int|y|^{p} d x \\
& =\frac{1}{2} \frac{\lambda_{k}-\lambda}{\lambda_{k}}\|y\|_{\mu}^{2}-\frac{1}{p}|y|_{p}^{p} .
\end{aligned}
$$

Thus $J_{\mu}(y) \leq 0$ since all norms are equivalent on $Y$. Let $e:=\phi_{k+1}$ be the $(k+1)$ th eigenfunction of $\left(-\triangle-\mu /|x|^{2}\right)$, since for any $y \in Y$,

$$
J_{\mu}\left(y+t \phi_{k+1}\right) \longrightarrow-\infty \quad \text { as } t \longrightarrow \infty .
$$

It follows from Remark 3.2 that we can take $T$ sufficiently large and define $Q=\left(\overline{B_{T}} \cap Y\right) \oplus$ $\{r e ; 0<t<T\}$ such that Proposition 3.4 holds.

The proof in the case of $c \geq \alpha$ is the same as in the proof of [5, Theorem 5.3], by now we have completed the proof of Theorem 1.1.

\section{Proof of Theorem 1.3}

In this section, we will prove Theorem 1.3. Here we define the following Euler-Lagrange functional of (1.3) on $H$ :

$$
\tilde{J}_{\mu}(u)=\frac{1}{2} \int\left(|\nabla u|^{2}-\frac{\mu}{|x|^{2}} u^{2}\right) d x-\frac{1}{p} \int\left(u^{+}\right)^{p} d x-\frac{\lambda}{2} \int\left(u^{+}\right)^{2} d x,
$$

where $u^{+}=\max \{u, 0\}$, and for any $\varphi \in C_{0}^{\infty}(\Omega)$,

$$
\left\langle\tilde{J}_{\mu}(u), \varphi\right\rangle=\int\left(\nabla u \nabla \varphi-\frac{\mu}{|x|^{2}} u \varphi\right) d x-\int\left(u^{+}\right)^{p-1} \varphi d x-\lambda \int\left(u^{+}\right) \varphi d x .
$$

By using the same method in the proof of Theorem 1.1, we obtain that $\tilde{J}_{\mu}$ satisfies (PS) condition. Next, we just use the mountain pass theorem to prove Theorem 1.3. 
It is easy to check that $\tilde{J}_{\mu}(u) \in C^{1}\left(H_{0}^{1}(\Omega), \mathbb{R}\right)$, we will verify the assumptions of the mountain pass theorem. By the Sobolev theorem, there exists $c_{1}>0$, such that for $u \in$ $H,\|u\|_{L^{p}(\Omega)} \leq c_{1}\|u\|_{\mu}$. Hence we have

$$
\begin{aligned}
\widetilde{J_{\mu}}(u) & =\frac{1}{2} \int\left(|\nabla u|^{2}-\frac{\mu}{|x|^{2}} u^{2}\right) d x-\frac{1}{p} \int\left(u^{+}\right)^{p} d x-\frac{\lambda}{2} \int\left(u^{+}\right)^{2} d x \\
& \geq \frac{1}{2}\|u\|_{\mu}^{2}-\frac{c_{1}}{p}\|u\|_{\mu}^{p}-\frac{\lambda}{2 \lambda_{1}}\|u\|_{\mu}^{2} \\
& =\frac{1}{2}\left(1-\frac{\lambda}{\lambda_{1}}\right)\|u\|_{\mu}^{2}-\frac{c_{1}}{p}\|u\|_{\mu}^{p} .
\end{aligned}
$$

So there is $r>0$ such that

$$
b:=\inf _{\|u\|_{\mu}=r} \tilde{J}_{\mu}(u)>0=\widetilde{J}_{\mu}(0)
$$

Let $u \in H$ with $u>0$ on $\Omega$, we have, for $t \geq 0$,

$$
\tilde{J_{\mu}}(t u)=\frac{t^{2}}{2} \int\left(|\nabla u|^{2}-\frac{\mu}{|x|^{2}} u^{2}\right) d x-\frac{t^{p}}{p} \int\left(u^{+}\right)^{p} d x-\frac{\lambda t^{2}}{2} \int\left(u^{+}\right)^{2} d x .
$$

Since $p>2$, there exists $e:=t u$, such that $\|e\|_{\mu}>r$ and $\tilde{J}_{\mu}(e) \leq 0$. By the mountain pass theorem, $\widetilde{J}_{\mu}$ has a positive critical value, and problem

$$
\begin{gathered}
-\Delta u-\frac{\mu}{|x|^{2}} u=\left(u^{+}\right)^{p-1}+\lambda u^{+} \quad \text { in } \Omega \backslash\{0\}, \\
u \in H_{0}^{1}(\Omega)
\end{gathered}
$$

has a nontrivial solution $u$. Multiplying the equation by $u^{-}$and integrating over $\Omega$, we find

$$
0=\int\left(\left|\nabla u^{-}\right|^{2}-\frac{\mu}{|x|^{2}}\left(u^{-}\right)^{2}\right) d x=\left\|u^{-}\right\|_{\mu^{*}}^{2}
$$

Hence $u^{-}=0$, that is, $u \geq 0$. A standard elliptic regularity argument implies that $u \in$ $C^{2}(\Omega \backslash\{0\})$, in which case, by the strong maximum principle, $u$ is positive, thus is the solution of problem (1.3).

\section{Acknowledgments}

The authors would like to thank Professor Zhaoli Liu for many valuable comments which improved the manuscript. The authors acknowledge the support of NNSF of China (10161010) and Fujian Provincial Natural Science Foundation of China (A0410015). 


\section{References}

[1] A. Capozzi, D. Fortunato, and G. Palmieri, An existence result for nonlinear elliptic problems involving critical Sobolev exponent, Annales de l'Institut Henri Poincaré. Analyse Non Linéaire 2 (1985), no. 6, 463-470.

[2] J. Chen, Existence of solutions for a nonlinear PDE with an inverse square potential, Journal of Differential Equations 195 (2003), no. 2, 497-519.

[3] J. P. García Azorero and I. Peral Alonso, Hardy inequalities and some critical elliptic and parabolic problems, Journal of Differential Equations 144 (1998), no. 2, 441-476.

[4] E. Jannelli, The role played by space dimension in elliptic critical problems, Journal of Differential Equations 156 (1999), no. 2, 407-426.

[5] P. H. Rabinowitz, Minimax Methods in Critical Point Theory with Applications to Differential Equations, CBMS Regional Conference Series in Mathematics, vol. 65, American Mathematical Society, Rhode Island, 1986.

[6] M. Willem, Minimax Theorems, Progress in Nonlinear Differential Equations and Their Applications, 24, Birkhäuser Boston, Massachusetts, 1996.

Shenghua Weng: Department of Mathematics, Fujian Normal University, Fuzhou 350007, China E-mail address: azhen1998@163.com

Yongqing Li: Department of Mathematics, Fujian Normal University, Fuzhou 350007, China E-mail address: yqli@fjnu.edu.cn 